Document downloaded from:

http://hdl.handle.net/10251/64856

This paper must be cited as:

Martinez, A.; Abasolo, D.; Alcaraz, R.; Rieta, JJ. (2015). Alteration of the P-wave non-linear dynamics near the onset of paroxysmal atrial fibrillation. Medical Engineering and Physics. 37(7):692-697. doi:10.1016/j.medengphy.2015.03.021

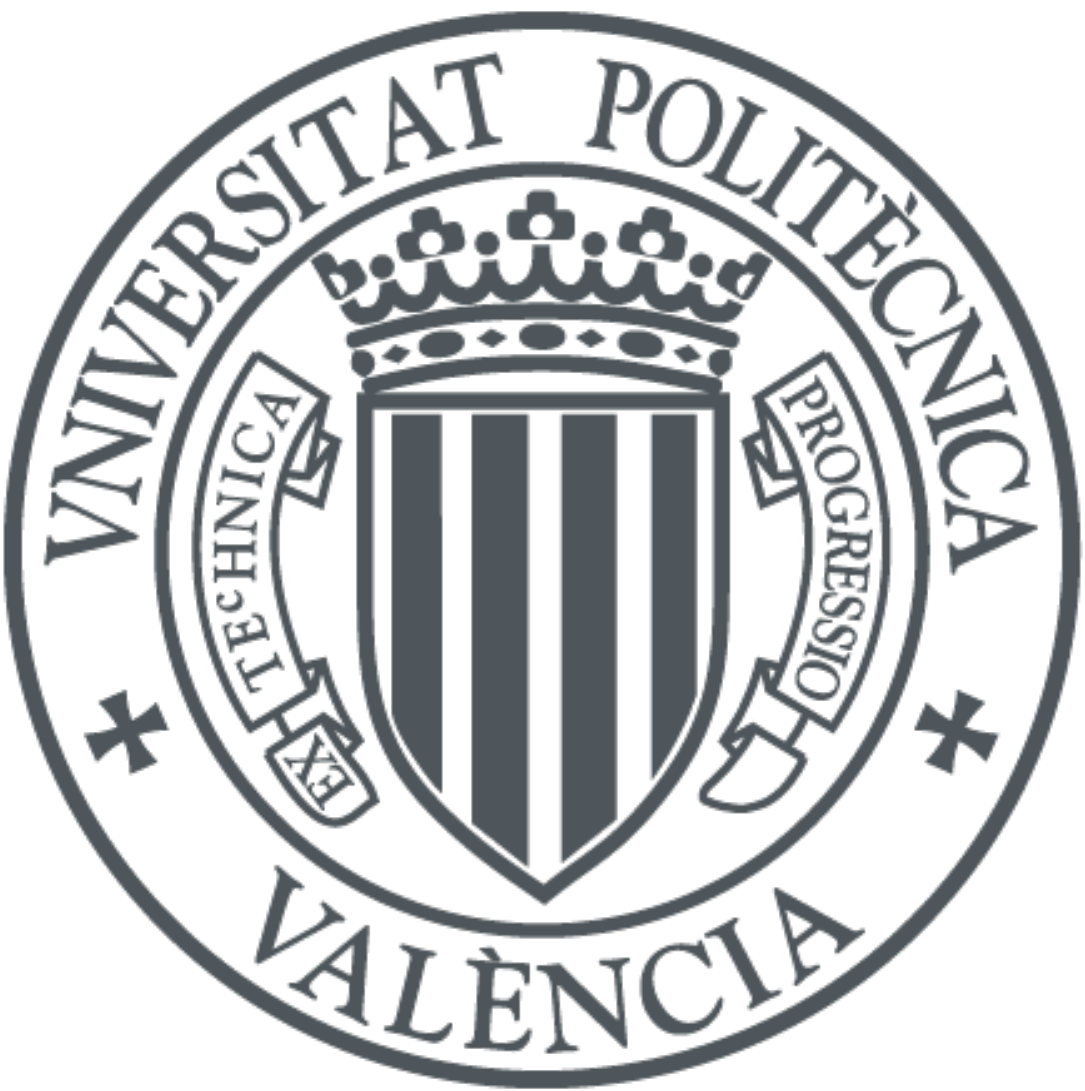

The final publication is available at

http://dx.doi.org/10.1016/j.medengphy.2015.03.021

Copyright Elsevier

Additional Information 


\title{
Alteration of the P-wave Non-linear Dynamics Near the Onset of Paroxysmal Atrial Fibrillation
}

\author{
Arturo Martínez ${ }^{1}$, Daniel Abásolo ${ }^{2}$, Raúl Alcaraz ${ }^{1}$, José J. Rieta ${ }^{3}$ \\ ${ }^{1}$ Innovation in Bioengineering Research Group, University of Castilla-La Mancha, Spain. \\ ${ }^{2}$ Centre for Biomedical Engineering, Department of Mechanical Engineering Sciences, University of Surrey, UK. \\ ${ }^{3}$ Biomedical Synergy, Electronic Engineering Department, Universidad Politécnica de Valencia, Spain
}

\begin{abstract}
The analysis of P-wave variability from the electrocardiogram (ECG) has been suggested as an early predictor of the onset of paroxysmal atrial fibrillation (PAF). Hence, a preventive treatment could be used to avoid the loss of normal sinus rhythm, thus minimising health risks and improving the patient's quality of life. In these previous studies the variability of different temporal and morphological P-wave features has been only analyzed in a linear fashion. However, the electrophysiological alteration occurring in the atria before the onset of PAF has to be considered as an inherently complex, chaotic and non-stationary process. This work analyses the presence of non-linear dynamics in the P-wave progression before the onset of PAF through the application of the central tendency measure (CTM), which is a non-linear metric summarising the degree of variability in a time series. Two hour-length ECG intervals just before the arrhythmia onset belonging to 46 different PAF patients were analysed. In agreement with the invasively observed inhomogeneous atrial conduction preceding the onset of PAF, CTM for all the considered P-wave features showed higher variability when the arrhythmia was closer to its onset. A diagnostic accuracy around $80 \%$ to discern between ECG segments far from PAF and close to PAF was obtained with the CTM of the metrics considered. This result was similar to previous P-wave variability methods based on linear approaches. However, the combination of linear and non-linear methods with a decision tree improved considerably their discriminant ability up to $90 \%$, thus suggesting that both dynamics could coexist at the same time in the fragmented depolarisation of the atria preceding the arrhythmia.
\end{abstract}

Keywords: Atrial fibrillation, Central tendency measure, Electrocardiogram, Non-linear, P-wave

Number of words: 3455

Address for correspondence:

Raúl Alcaraz Martínez, Escuela Politécnica, Campus Universitario, 16071, Cuenca, Spain.

Phone: +34-969-179-100 Ext. 4847; Fax: +34-969-179-119.

e-mail: raul.alcaraz@uclm.es 


\section{Introduction}

Atrial fibrillation (AF) is the most common sustained supra-ventricular arrhythmia in clinical practice, with an increasing number of patients being affected worldwide [1]. From a clinical point of view, this arrhythmia can present itself in different forms [2]. It often starts with paroxysmal (self-terminating) episodes, which have a duration ranging from several seconds to less than 7 days. Previous studies have estimated a progression rate of PAF patients to persistent or permanent AF between 15 and $31 \%$, this transition occurring during a time period of 4 to 8 years, approximately [3]. Both persistent and permanent AF episodes last more than 7 days, their main difference being the dissimilar response to cardioversion, which has only proven to be effective in persistent AF.

Nowadays, the mechanisms provoking the onset of PAF are not fully known [4]. However, significant heterogeneous alterations in atrial electrophysiological properties have been noticed before the spontaneous onset of the arrhythmia [5]. Such abnormalities may result in an anisotropic and discontinuous propagation of sinus impulses, thus predisposing the atria to fibrillation [5]. This uncoordinated atrial activity reaches the atrio-ventricular (AV) node and can be conducted through the ventricles, which leads to a fast and irregular heart rate [1]. Even though PAF is self-limited, its presence can provoke major complications such as decreased heart capacity, thromboembolic events, congestive heart failure, and tachycardia-induced cardiomyopaty [6]. Consequently, although PAF is not a life-threatening arrhythmia, it increases cardiovascular morbidity and mortality [7]. Therefore, after the spontaneous termination of a PAF episode, a very relevant clinical challenge starts: to predict the arrival of the next episode. The possibility of avoiding PAF recurrence by using early pacing and drugs could hamper the progression of PAF to a more chronic form $[1,3]$.

Recently, the analysis of P-wave variability over time has shown a certain ability to quantify electrophysiological alterations during the two hours preceding the onset of PAF $[8,9]$. In these studies, every P-wave was delineated and then characterised by computing temporal distances between its fiducial points [8] or quantifying its morphology [9]. Next, the variability evolution of each analysed parameter was estimated by means of a linear fitting $[8,9]$. However, the possible presence of nonlinear dynamics within the P-wave evolution before the onset of PAF was not studied. To this respect, 
the study of the P-wave variability over time from a non-linear point of view could reveal clinically interesting information and complement these previous works. In fact, atrial electrophysiological evolution preceding the onset of PAF can be considered as an inherently chaotic and non-stationary process [5,10], which could be modelled by non-linear equations [11,12].

The present study hypothesises that the P-wave variability estimation over time through nonlinear methods may help to anticipate recurrent PAF episodes which could be misdiagnosed by linear methods previously proposed $[8,9]$. To this respect, non-linear methods have previously demonstrated their interesting capabilities dealing with the envision of events related to AF [13,14], as well as in tracking the behaviour of this arrhythmia [15]. Moreover, through the combination of both linear and non-linear approaches, PAF onset prediction may be improved. To the best of our knowledge, nonlinear analysis of P-wave variability has never been applied to quantify susceptibility to PAF. In the present work, the central tendency measure (CTM) will be computed to analyse the alteration of the P-wave non-linear dynamics. This index uses continuous chaotic modelling to summarise the degree of variability in a time series [16].

\section{Methods}

\subsection{Study population}

A cohort of 46 patients with idiopathic PAF, i.e. none of them caused by concomitant heart disease, hyperthyroidism or pulmonary disease, were retrospectively selected for the study. Their main demographic and clinical characteristics are presented in Table 1. At the time of the study any patient was under anti-arrhythmic drug therapy. Further details on the database are to be found elsewhere [9]. The study was approved by the hospital's Ethics Committee and informed consent was obtained from all the participants. 


\subsection{P-wave delineation and characterisation}

A 24-h Holter recording was acquired from every patient. A sampling rate of $1000 \mathrm{~Hz}$ and 16-bit resolution were considered as recording parameters. Next, the longest sinus rhythm interval was selected and the two hours before the onset of PAF were extracted and divided into two-one hour periods. This division aimed to evaluate how the proposed approach quantified the P-wave non-linear variability over time. The ECG segments immediately before and further than the arrhythmia onset were named as "close to PAF" and "far from PAF", respectively.

After the ECGs segmentation, P-waves were detected from lead V1 and their boundaries automatically delineated [17]. Although more recent approaches for this purpose can be found in the literature (e.g. $[18,19])$, the algorithm used here provided high accuracy and very few location errors [17]. Moreover, it is worth noting that automatic P-wave delineation allows to obtain highly reproducible measurements [20]. Nonetheless, expert cardiologists supervised the obtained P-wave detection and delineation and around $4 \%$ of the P-waves had to be corrected.

Finally, each P-wave was characterised by a set of morphological and time features. Morphological changes provoked by alterations in the atrial depolarisation were first quantified by three parameters previously analysed [9]. Thus, the rectified P-wave length was computed as its arc length $\left(\mathrm{P}_{\mathrm{al}}\right)$ and the $\mathrm{P}$-wave amplitude was characterised by its normalised root mean square (nrms) value $\left(\mathrm{P}_{\text {nrms }}\right)$ and its area $\left(\mathrm{P}_{\text {area }}\right)$. On the other hand, different temporal features related to the $\mathrm{P}$-wave fiducial points have also proven to be indicative of an increased risk of AF development $[4,8]$. Hence, the $\mathrm{P}$-wave duration $\left(\mathrm{P}_{\text {dur }}\right)$ together with the duration of its initial $\left(\mathrm{P}_{\text {ini }}\right)$ and terminal $\left(\mathrm{P}_{\text {ter }}\right)$ portions were computed. The rhythm variability between successive P-waves was also estimated (PP) [8].

\subsection{Central tendency measure}

In order to quantify the P-wave non-linear features variability over time, CTM was computed from the time series generated by each single parameter and calculated in a wave-to-wave fashion. CTM is a quantitative measure of variability computed from second-order difference plots [16]. Given a time series $x[n]$, the second-order difference plot, i.e., the graph $x[n+2]-x[n+1]$ versus $x[n+1]-x[n]$, 
centred around the origin represents graphically its rate of variability [16]. Thus, by selecting a circular region of radius $\rho$ around the origin, CTM is computed by counting the number of points that fall within the radius and dividing by the total number of points. In this way, a low CTM value indicates a large amount of dispersion and a high value indicates concentration near the centre.

The outcome of CTM for a concrete application depends strongly on the selected radius. However, in contrast to other non-linear methods [21], no guidelines exist for optimising its value. Hence, it is usually chosen depending upon the character of the data [16]. In the present study an approach similar to the developed in previous works $[22,23]$ was used to select the optimal $\rho$ for each single P-wave feature. Thus, CTM was computed for radius of $0.1,0.2, \ldots, 10$ times the standard deviation of the analysed data. Normalising $\rho$ in this way provides translation and scale invariance, in the sense that CTM remains unchanged under uniform process magnification, reduction or constant shift to higher or lower values. Thereafter, for each considered $\rho$, statistical differences between CTM values for ECG segments far from PAF and close to PAF were assessed by a Student's $t$-test or a U MannWhitney test depending on the normality and homoscedasticity of the data, respectively. To evaluate these conditions, the Kolmogorov-Smirnov and Levene tests were used, respectively. Finally, the selected radius was determined as the one providing the lowest statistical significance ( $p$-value).

\subsection{Linear estimation of P-wave variability}

To better contextualising the results obtained by CTM, the P-wave variability over time was also estimated in a linear fashion from the three most significant metrics reported in previous studies, i.e. $\mathrm{P}_{\mathrm{dur}}, \mathrm{P}_{\mathrm{al}}$ and PP [8,9]. In brief, groups of 10 samples were formed from the data series for each parameter. Then, for every group the difference between its 90- and 10-quantiles was obtained to estimate its variability. Finally, the groups variability time course for each parameter, i.e. their slope $\alpha$, was computed from a linear least-squares fitting. 


\subsection{Performance assessment}

A stratified 2-fold cross-validation was used to assess the ability of CTM and the slope $\alpha$ computed from each single metric in discerning between ECG segments far from PAF and close to PAF. In this approach, the database is randomly partitioned into two equal size subsamples. For each fold, approximately the same number of ECG segments from each group is considered. Then, a single subsample is used for training and the other one for testing. The process is then repeated changing the role of each fold. Finally, classification results are separately averaged for both iterations. In order to assess the discriminant ability of each parameter, a receiver operating characteristic (ROC) curve was used in each training process. The rate of ECG segments far from PAF properly identified was considered as the true positive rate (i.e., sensitivity). Similarly, the percentage of ECG segments close to PAF successfully classified were considered as the true negative rate (i.e., specificity). The optimum threshold discriminating both groups was finally selected as the CTM or $\alpha$ value providing the highest accuracy, i.e., the greatest rate of ECG segments correctly discerned.

Additionally, the relationships among the variability estimated from single P-wave parameters through CTM and the slope $\alpha$ were analysed by means of a decision tree. Thus, the optimal combination of CTM computed from every single parameter with the slope $\alpha$ obtained from $\mathrm{P}_{\mathrm{dur}}, \mathrm{P}_{\mathrm{al}}$ and PP was analysed. The tree growth was stopped when each node only contained ECGs from a group or less than $20 \%$ of all ECGs. Moreover, every node was split by using an impurity-based Gini index [24]. A stratified 2-fold cross-validation was also used to evaluate the classification result of each tree.

\section{Results}

The optimal radius $\rho$ providing the highest statistical differences between ECG segment groups as well as the mean and standard deviation of the computed CTM values are shown in Table 2. As can be observed, all the metrics provided statistically significant differences between groups, revealing higher mean values for ECG segments far from PAF, such as Fig. 1 shows for $\mathrm{P}_{\mathrm{al}}, \mathrm{P}_{\mathrm{dur}}$ and $\mathrm{P}_{\text {ini }}$. As another significant example, Fig. 2 shows the second-order difference plot associated to $\mathrm{P}_{\text {dur }}$ for a 
typical patient, presenting higher dispersion in ECG segments close to PAF. In contrast, the slope $\alpha$ for $\mathrm{P}_{\mathrm{dur}}, \mathrm{P}_{\mathrm{al}}$ and PP presented an increase in the mean values associated to ECG segments close to PAF, such as shown in Fig. 3. Anyway, statistically significant differences were also found between groups.

In order to estimate the diagnostic accuracy of each studied metric in a more robust way, the stratified 2-fold cross-validation was run five times. Thus, Tables 3 and 4 present the average values of sensitivity, specificity and accuracy obtained for the 10 iterations of learning and test carried out from the CTM and $\alpha$ values, respectively. Regarding CTM, the highest classification rates (around 80\%) were achieved with the metrics $\mathrm{P}_{\mathrm{dur}}, \mathrm{P}_{\mathrm{ini}}$ and $\mathrm{P}_{\mathrm{al}}$, such as Table 3 shows. However, as can be seen from Table 4, the slope $\alpha$ from $\mathrm{P}_{\mathrm{al}}$ and $\mathrm{P}_{\mathrm{dur}}$ reached accuracy values slightly higher than $80 \%$.

Finally, a decision tree modelled by two metrics was obtained for every 4-parameter subset constituted by the CTM, corresponding to one of the studied P-wave features, and the slope $\alpha$ for $\mathrm{P}_{\mathrm{al}}$, $\mathrm{P}_{\mathrm{dur}}$ and PP. As can be observed in Table 5, the slope $\alpha$ for $\mathrm{P}_{\mathrm{al}}$ was the most frequently chosen parameter to complement the CTM. Moreover, for all the metrics excepting $\mathrm{P}_{\text {ter }}$ and PP, the decision tree results outperformed the slope $\alpha$ for $\mathrm{P}_{\mathrm{al}}$, reaching accuracy values around $87 \%$. Nonetheless, it is worth noting that for every generated decision tree the combination of the same two P-wave metrics remained unaltered from the 10 learning iterations in the cross-validation analysis. To this respect, Fig. 4 shows the tree structure obtained for one of the learning iterations from the combination of CTM for $\mathrm{P}_{\text {ini }}$ and the slope $\alpha$ for $\mathrm{P}_{\mathrm{al}}$. As can be seen, ECG segments far from PAF were identified by the lowest degrees of both linear and non-linear variability.

\section{Discussion}

According to the intermittently disturbed conduction observed in the atrial tissue susceptible to PAF [5], the present study has shown that CTM is able to quantify the P-wave variability progression over the two hours preceding the onset of PAF. To this respect, a higher variability was observed as the arrhythmia onset approximates, thus suggesting that non-linear dynamics exist in the transition from sinus rhythm to PAF. This finding agrees with previous works demonstrating that steep conduction 
velocity dispersion represents one way to form a spatially heterogeneous pattern in a completely homogeneous tissue [25], the theory of such pattern formation being a well-described part of non-linear systems theory [11].

Compared to the P-wave variability linear estimation, CTM did not improve PAF onset prediction. Thus, the two highest classification rates were obtained by the slope $\alpha$ for $\mathrm{P}_{\mathrm{al}}$ and $\mathrm{P}_{\mathrm{dur}}$, respectively. Nonetheless, CTM for the same P-wave features reported a slightly lower diagnostic accuracy only worsened by less than 3\%. A similar result was also observed for CTM of the P-wave initial portion, i.e., $\mathrm{P}_{\text {ini }}$. Anyway, it has to be remarked that the determination of CTM requires a notable lower computational burden than a linear least-squares fitting. Indeed, only comparisons between points and one division are required to compute CTM, whereas numerous additions and multiplications are used in a linear regression. Therefore, CTM could be more easily implemented on real-time ECG monitoring systems. On the other hand, the combination of linear and non-linear estimates by a decision tree improved considerably the diagnostic accuracy of every single parameter, thus validating our initial hypothesis. Moreover, this result also suggests that the P-wave variability before the onset of PAF is a complex process, in which linear and non-linear dynamics coexist simultaneously within the disrupted atrial depolarisation.

PAF onset prediction has also been addressed in several previous studies. Most of them have analysed the RR series or the atrial premature contractions (APCs) preceding the arrhythmia. These studies were mainly performed within the Computers in Cardiology (CinC) Challenge 2001 and made use of a database designed for that purpose [26] freely available at PhysioNet [27]. Although this database contained 53 patients, it presented serious disadvantages. First, no clinical data were provided by PhysioNet. Therefore, the effect of confounding factors such as age, gender or heart rate on P-wave features [28] cannot be controlled in the study. Second, the ECGs were sampled at $128 \mathrm{~Hz}$, which hinders an accurate P-wave characterisation [29]. Finally, only 30 minute-length ECG intervals were provided, thus turning impossible the analysis of longer time intervals before PAF onset, such as the presented in this study.

Nonetheless, with the aim to compare the proposed algorithm with previous works, the CinC's Challenge 2001 database was also used. More precisely, the 28 test recording sets provided for the 
so called "Event 2" were analysed. Each set contained two recordings, one immediately prior to an episode of PAF and another distant ( $\geq 45$ minutes) from any such episode, the event consisting in the identification of which segment immediately preceded PAF. The proposed algorithm reported a score of $93 \%$ (26 out of 28), thus improving all the methods presented at CinC's Challenge, because no scores higher than 79\% (22 out of 28) were reported [26]. Additionally, this result outperforms later works, such as the one reported by Thong et al [30], who reached a score of $89 \%$ (25 out of 28) by studying the number of APCs and the subsequent rhythms in the RR series. Others have also studied the same database, but without respecting its organisation. Thus, learning and test sets were taken together and no statistical validation of the results was considered in later works $[31,32]$. In these cases, classification rates higher than $90 \%$ were reached. However, a wide variety of time, frequency and complexity features of the RR series were combined through highly complex classifiers [31,32], thus hindering the clinical interpretation of each single parameter within the predictive model. In contrast to these works, the proposed tree-based model yielded a comparable diagnostic accuracy by just combining two parameters, thus making the clinical understading of its outcomes easier. To this respect, the higher the P-wave variability estimated both by the linear and non-linear methods, the higher the risk of an early onset of PAF.

On the other hand, some of these previous works also tried to quantify the electrophysiological alterations preceding the onset of PAF. Thus, a decrease of complexity indices and a increase in the spectral energy of the RR dynamics some minutes before the onset of PAF have been reported [33,34]. Similarly, the detection of a high number of APCs has proven to be a good harbinger of the imminent onset of PAF [30]. However, this high concentration of APCs only happens some minutes before the onset $[33,34]$. As a consequence, these previous works can anticipate a PAF episode only few minutes before its onset, thus making the administration of a feasible antiarrythmic drug treatment impossible. In contrast, CTM has revealed the ability to identify atrial alterations and anticipate a PAF episode at least two hours before its onset.

Finally, several study limitations have to be mentioned. First, a reduced database was only analysed, thus further prospective studies would be needed to confirm the proposed methodology robustness as well as obtained results reproducibility. Second, the earliest mark of PAF onset has not been 
determined because the two hours preceding the arrhythmia were only considered. Thus, further analysis leading to reach this significant event have to be developed. Finally, standard lead V1 was only studied, thus rejecting potential information contained in other leads.

\section{Conclusions}

Alteration of the temporal and morphological P-wave non-linear dynamics over time has been successfully quantified through the CTM, noticing higher variability in the P-wave features when the arrhythmia was closer to its onset. This P-wave non-linear variability analysis has also reported a similar diagnostic accuracy than its linear counterpart in the discrimination between ECG segments far from PAF and close to PAF. However, the computational cost of the proposed non-linear CTMbased analysis is notably lower. Finally, the combination of both linear and non-linear estimates of P-wave variability through a decision tree has improved significantly the discrimination ability, thus suggesting that linear and non-linear dynamics coexist at the same time in the disturbed atrial depolarisation preceding the onset of PAF.

\section{Acknowledgements}

This work was supported by the projects TEC2014-52250-R from the Spanish Ministry of Economy Competitiveness and PPII-2014-026-P from Junta de Comunidades de Castilla-La Mancha.

\section{Declarations}

Competing interests: None declared.

Funding: This work was supported by the projects TEC2014-52250-R from the Spanish Ministry of Economy Competitiveness and PPII-2014-026-P from Junta de Comunidades de Castilla-La Mancha.

Ethical approval: The study was approved by our hospital's Ethics Committee and informed consent was obtained from all the participants. 


\section{References}

[1] Wann LS, Curtis AB, Ellenbogen KA, Estes NAM, Ezekowitz MD, Jackman WM, et al. Management of patients with atrial fibrillation (compilation of 2006 ACCF/AHA/ESC and 2011 ACCF/AHA/HRS recommendations): a report of the American College of Cardiology/American Heart Association Task Force on practice guidelines. Circulation 2013: 127(18):1916-26.

[2] Gallagher MM and Camm J. Classification of atrial fibrillation. Am J Cardiol 1998: $82(8 \mathrm{~A}): 18 \mathrm{~N}-28 \mathrm{~N}$.

[3] de Vos CB, Pisters R, Nieuwlaat R, Prins MH, Tieleman RG, Coelen RJS, et al. Progression from paroxysmal to persistent atrial fibrillation clinical correlates and prognosis. J Am Coll Cardiol 2010:55(8):725-31.

[4] Platonov PG. P-wave morphology: underlying mechanisms and clinical implications. Ann Noninvasive Electrocardiol 2012:17(3):161-9.

[5] Dilaveris PE and Gialafos JE. P-wave dispersion: a novel predictor of paroxysmal atrial fibrillation. Ann Noninvasive Electrocardiol 2001:6(2):159-65.

[6] Thrall G, Lane D, Carroll D, and Lip GYH. Quality of life in patients with atrial fibrillation: a systematic review. Am J Med 2006:119(5):448.e1-19.

[7] Stewart S, Hart CL, Hole DJ, and McMurray JJV. A population-based study of the long-term risks associated with atrial fibrillation: 20-year follow-up of the Renfrew/Paisley study. Am J Med 2002:113(5):359-64.

[8] Martínez A, Alcaraz R, and Rieta JJ. Study on the P-wave feature time course as early predictors of paroxysmal atrial fibrillation. Physiol Meas 2012:33(12):1959-74.

[9] Martínez A, Alcaraz R, and Rieta JJ. Morphological variability of the P-wave for premature envision of paroxysmal atrial fibrillation events. Physiol Meas 2014:35(1):1-14. 
[10] Qu Z. Chaos in the genesis and maintenance of cardiac arrhythmias. Prog Biophys Mol Biol 2011:105(3):247-57.

[11] Karma A and Gilmour RF. Nonlinear dynamics of heart rhythm disorders. Physics Today 2007: 60:51-57.

[12] Krogh-Madsen T and Christini DJ. Nonlinear dynamics in cardiology. Annu Rev Biomed Eng 2012:14:179-203.

[13] Alcaraz R and Rieta JJ. A non-invasive method to predict electrical cardioversion outcome of persistent atrial fibrillation. Med Biol Eng Comput 2008:46(7):625-35.

[14] Alcaraz R and Rieta JJ. A novel application of sample entropy to the electrocardiogram of atrial fibrillation. Nonlinear Analysis: Real World Applications 2010:11(2):1026-1035.

[15] Alcaraz R and Rieta JJ. Non-invasive organization variation assessment in the onset and termination of paroxysmal atrial fibrillation. Comput Methods Programs Biomed 2009:93(2):148-54.

[16] Cohen M, Hudson D, and Deedwania P. Applying continuous chaotic modeling to cardiac signals. IEEE Eng Med Biol Mag 1996:15:97-102.

[17] Martínez A, Alcaraz R, and Rieta JJ. Application of the phasor transform for automatic delineation of single-lead ECG fiducial points. Physiol Meas 2010:31(11):1467-85.

[18] Saini I, Singh D, and Khosla A. K-nearest neighbour-based algorithm for P- and T-waves detection and delineation. J Med Eng Technol 2014:38(3):115-24.

[19] Homaeinezhad MR, Erfanianmoshiri-Nejad M, and Naseri H. A correlation analysis-based detection and delineation of ECG characteristic events using template waveforms extracted by ensemble averaging of clustered heart cycles. Comput Biol Med 2014:44:66-75.

[20] Clavier L, Boucher JM, Lepage R, Blanc JJ, and Cornily JC. Automatic P-wave analysis of patients prone to atrial fibrillation. Med Biol Eng Comput 2002:40(1):63-71. 
[21] Alcaraz R, Abásolo D, Hornero R, and Rieta JJ. Optimal parameters study for sample entropybased atrial fibrillation organization analysis. Comput Methods Programs Biomed 2010: 99(1):124-32.

[22] Gutiérrez-Tobal GC, Hornero R, Alvarez D, Marcos JV, and del Campo F. Linear and nonlinear analysis of airflow recordings to help in sleep apnoea-hypopnoea syndrome diagnosis. Physiol Meas 2012:33(7):1261-75.

[23] Thuraisingham RA. A classification system to detect congestive heart failure using second-order difference plot of RR intervals. Cardiol Res Pract 2009:2009:807379.

[24] Breiman L. Classification and regression trees. Cole Advanced Books and Software, 1984.

[25] Qu Z, Garfinkel A, Chen PS, and Weiss JN. Mechanisms of discordant alternans and induction of reentry in simulated cardiac tissue. Circulation 2000:102(14):1664-70.

[26] Moody G, Goldberger A, McClennen S, and Swiryn S. Predicting the onset of paroxysmal atrial fibrillation: The Computers in Cardiology Challenge 2001. Computers in Cardiology 2001:28:113-116.

[27] Goldberger AL, Amaral LA, Glass L, Hausdorff JM, Ivanov PC, Mark RG, et al. PhysioBank, PhysioToolkit, and PhysioNet: components of a new research resource for complex physiologic signals. Circulation 2000:101(23):E215-20.

[28] Dhala A, Underwood D, Leman R, Madu E, Baugh D, Ozawa Y, et al. Signal-averaged P-wave analysis of normal controls and patients with paroxysmal atrial fibrillation: a study in gender differences, age dependence, and reproducibility. Clin Cardiol 2002:25(11):525-31.

[29] Censi F, Calcagnini G, Corazza I, Mattei E, Triventi M, Bartolini P, et al. On the resolution of ECG acquisition systems for the reliable analysis of the P-wave. Physiol Meas 2012:33(2):N117.

[30] Thong T, McNames J, Aboy M, and Goldstein B. Prediction of paroxysmal atrial fibrillation by analysis of atrial premature complexes. IEEE Trans Biomed Eng 2004:51(4):561-9. 
[31] Chesnokov YV. Complexity and spectral analysis of the heart rate variability dynamics for distant prediction of paroxysmal atrial fibrillation with artificial intelligence methods. Artif Intell Med 2008:43(2):151-65.

[32] Mohebbi M and Ghassemian H. Prediction of paroxysmal atrial fibrillation based on non-linear analysis and spectrum and bispectrum features of the heart rate variability signal. Comput Methods Programs Biomed 2012:105(1):40-9.

[33] Vikman S, Mäkikallio TH, Yli-Mäyry S, Pikkujämsä S, Koivisto AM, Reinikainen P, et al. Altered complexity and correlation properties of R-R interval dynamics before the spontaneous onset of paroxysmal atrial fibrillation. Circulation 1999:100(20):2079-84.

[34] Shin DG, Yoo CS, Yi SH, Bae JH, Kim YJ, Park JS, et al. Prediction of paroxysmal atrial fibrillation using nonlinear analysis of the R-R interval dynamics before the spontaneous onset of atrial fibrillation. Circ J 2006:70(1):94-9. 

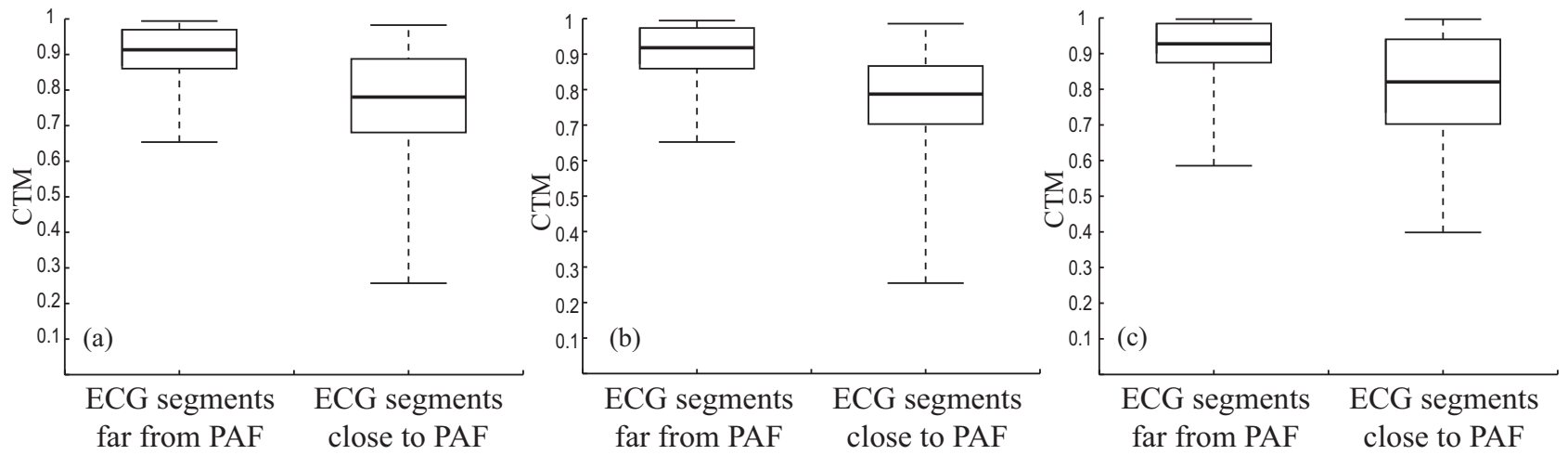

Figure 1: Boxplots of the CTM values computed from the most statistically significant features, i.e. (a) $\mathrm{P}_{\mathrm{al}}$, (b) $\mathrm{P}_{\text {dur }}$ and (c) $\mathrm{P}_{\text {ini }}$. 

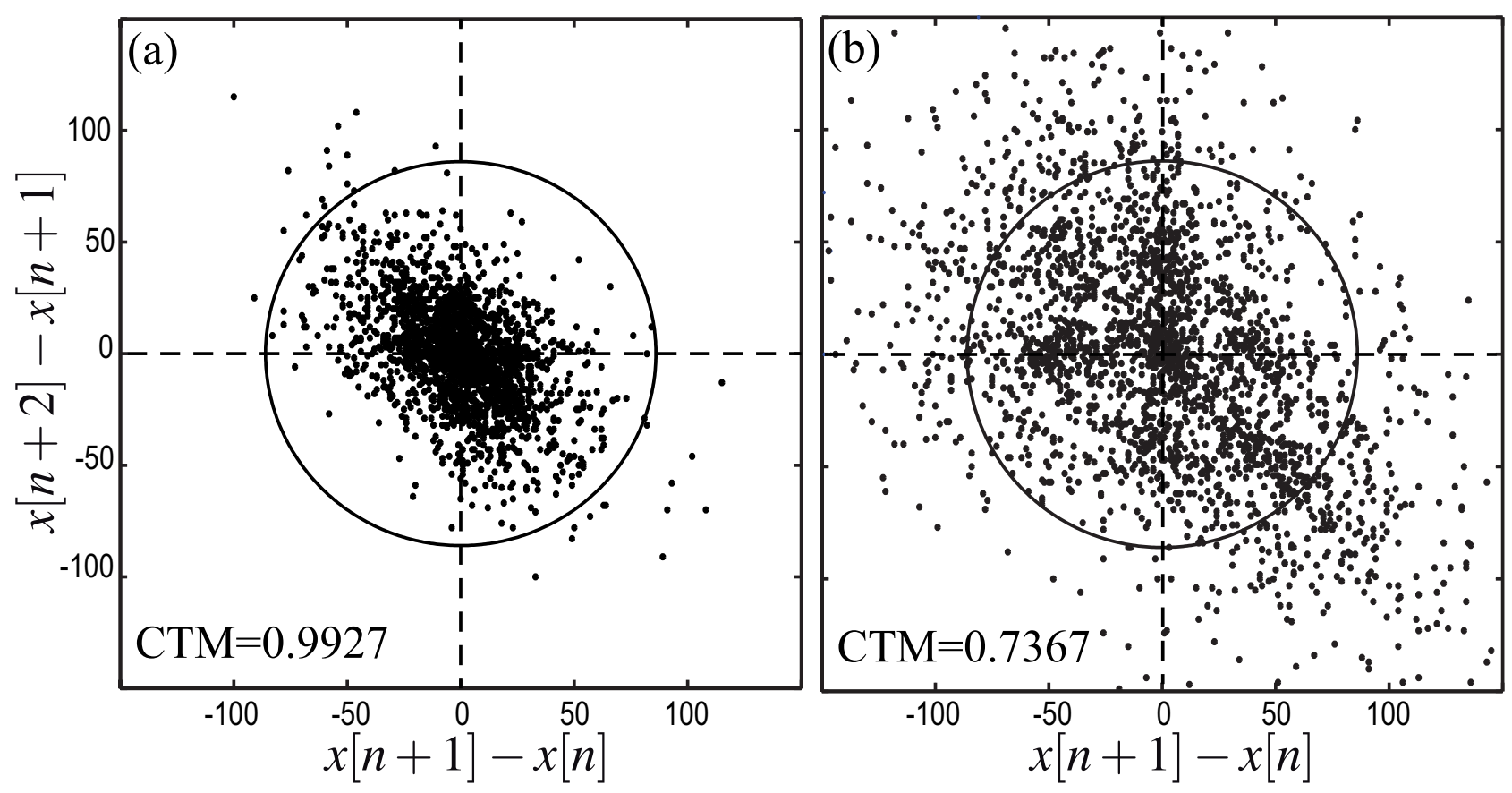

Figure 2: Second-order difference plot of the P-wave duration time course variability from a typical patient with segments (a) far from PAF and (b) close to PAF. 

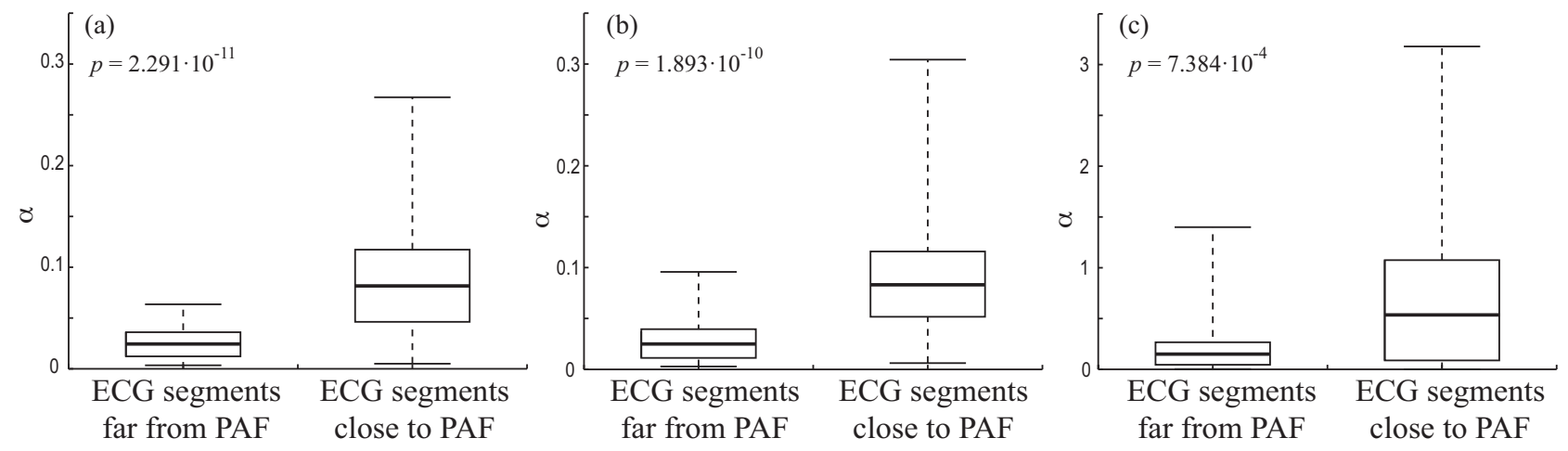

Figure 3: Boxplots of the slope $\alpha$ values computed from (a) $\mathrm{P}_{\mathrm{dur}}$, (b) $\mathrm{P}_{\mathrm{al}}$ and (c) PP. The statistical significance $p$ was obtained using a Student's $t$-test. 


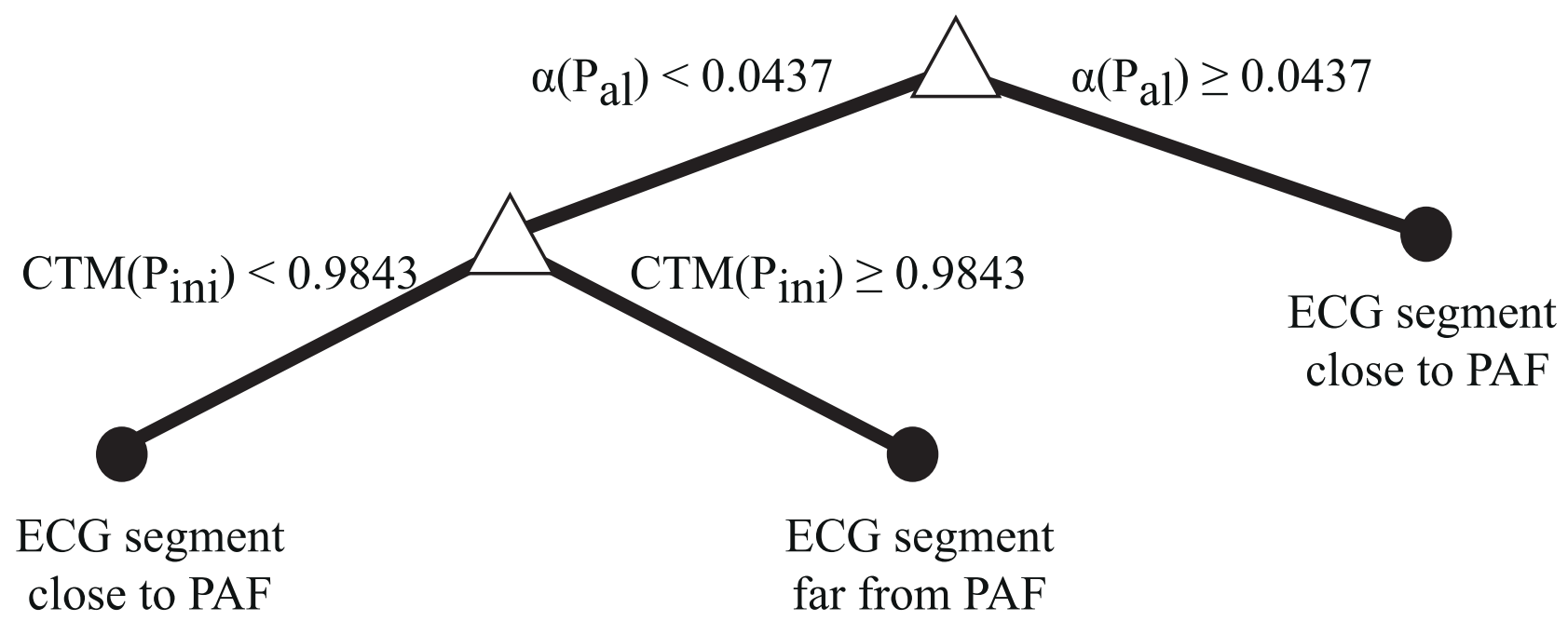

Figure 4: Combination of CTM for $\mathrm{P}_{\text {ini }}$ and the slope $\alpha$ for $\mathrm{P}_{\mathrm{al}}$ by means of a decision tree classifier in a training iteration. 
Table 1: Baseline demographic and clinical characteristics of the studied PAF patients.

\begin{tabular}{lc}
\hline Age (years) & $63.2 \pm 10.2$ \\
Gender (Male/Female) & $18 / 28$ \\
Height $(\mathrm{m})$ & $1.68 \pm 0.11$ \\
Weight $(\mathrm{kg})$ & $69.3 \pm 9.8$ \\
Heart rate (beats/min) & $68.4 \pm 7.1$ \\
Respiratory rate (breaths/min) & $13 \pm 1$ \\
QRS duration (ms) & $82.3 \pm 20.2$ \\
PQ duration (ms) & $164.1 \pm 1.6$ \\
\hline
\end{tabular}


Table 2: CTM values computed in average from the ECG segments far from PAF and close to PAF.

\begin{tabular}{lcccc}
\hline Feature & Optimal $\rho$ & Far from PAF & Close to PAF & $p$-value \\
\hline $\mathrm{P}_{\mathrm{al}}$ & 8.2 & $0.913 \pm 0.073$ & $0.780 \pm 0.179$ & $2.671 \times 10^{-8 \dagger}$ \\
$\mathrm{P}_{\text {nrms }}$ & 6.1 & $0.985 \pm 0.036$ & $0.942 \pm 0.093$ & $7.022 \times 10^{-5} \dagger$ \\
$\mathrm{P}_{\text {area }}$ & 6.3 & $0.985 \pm 0.025$ & $0.918 \pm 0.110$ & $4.771 \times 10^{-6 \dagger}$ \\
$\mathrm{P}_{\text {dur }}$ & 8.5 & $0.916 \pm 0.070$ & $0.787 \pm 0.177$ & $1.524 \times 10^{-7 \dagger}$ \\
$\mathrm{P}_{\text {ini }}$ & 8.3 & $0.927 \pm 0.084$ & $0.821 \pm 0.162$ & $1.301 \times 10^{-8 \dagger}$ \\
$\mathrm{P}_{\text {ter }}$ & 7.9 & $0.935 \pm 0.103$ & $0.836 \pm 0.179$ & $6.852 \times 10^{-6} \dagger$ \\
$\mathrm{PP}$ & 9.1 & $0.941 \pm 0.101$ & $0.876 \pm 0.139$ & $2.307 \times 10^{-3} \ddagger$ \\
\hline
\end{tabular}

$\dagger$ Statistical significance computed with the U Mann-Whitney test

\$ Statistical significance computed with the Student's $t$-test 
Table 3: Classification results provided by the CTM values computed from each analysed P-wave feature. Average values of sensitivity (Se), specificity (Sp) and accuracy (Acc) for the 10 iterations of learning and test carried out are presented.

\begin{tabular}{lccccccc}
\hline \multirow{2}{*}{ Feature } & \multicolumn{3}{c}{ Learning sets } & & \multicolumn{3}{c}{ Test sets } \\
\cline { 2 - 3 } & $\mathrm{Se}$ & $\mathrm{Sp}$ & $\mathrm{Acc}$ & & $\mathrm{Se}$ & $\mathrm{Sp}$ & $\mathrm{Acc}$ \\
\hline $\mathrm{P}_{\mathrm{al}}$ & $78 \%$ & $82 \%$ & $80 \%$ & & $80 \%$ & $79 \%$ & $80 \%$ \\
$\mathrm{P}_{\text {nrms }}$ & $66 \%$ & $71 \%$ & $68 \%$ & & $66 \%$ & $59 \%$ & $63 \%$ \\
$\mathrm{P}_{\text {area }}$ & $79 \%$ & $67 \%$ & $73 \%$ & $65 \%$ & $79 \%$ & $72 \%$ \\
$\mathrm{P}_{\text {dur }}$ & $80 \%$ & $78 \%$ & $79 \%$ & $76 \%$ & $83 \%$ & $80 \%$ \\
$\mathrm{P}_{\text {ini }}$ & $82 \%$ & $76 \%$ & $79 \%$ & $78 \%$ & $81 \%$ & $79 \%$ \\
$\mathrm{P}_{\text {ter }}$ & $74 \%$ & $82 \%$ & $78 \%$ & $73 \%$ & $68 \%$ & $71 \%$ \\
$\mathrm{PP}$ & $61 \%$ & $71 \%$ & $66 \%$ & $65 \%$ & $57 \%$ & $61 \%$ \\
\hline
\end{tabular}


Table 4: Classification results provided by the $\alpha$ values computed from $\mathrm{P}_{\mathrm{dur}}, \mathrm{P}_{\mathrm{al}}$ and PP. Mean values of sensitivity (Se), specificity (Sp) and accuracy (Acc) for the 10 iterations of learning and test carried out are presented.

\begin{tabular}{lccccccc}
\hline \multirow{2}{*}{ Feature } & \multicolumn{3}{c}{ Learning sets } & & \multicolumn{3}{c}{ Test sets } \\
\cline { 2 - 3 } \cline { 7 - 8 } & $\mathrm{Se}$ & $\mathrm{Sp}$ & $\mathrm{Acc}$ & & $\mathrm{Se}$ & $\mathrm{Sp}$ & Acc \\
\hline $\mathrm{P}_{\mathrm{dur}}$ & $84 \%$ & $90 \%$ & $87 \%$ & $79 \%$ & $85 \%$ & $82 \%$ \\
$\mathrm{P}_{\mathrm{al}}$ & $87 \%$ & $87 \%$ & $87 \%$ & $81 \%$ & $85 \%$ & $83 \%$ \\
$\mathrm{PP}$ & $73 \%$ & $75 \%$ & $74 \%$ & $65 \%$ & $71 \%$ & $68 \%$ \\
\hline
\end{tabular}


Table 5: Classification results provided by the decision tree obtained for each 4-feature subset constituted by CTM for one P-wave parameter and the slope $\alpha$ for $\mathrm{P}_{\mathrm{al}}, \mathrm{P}_{\mathrm{dur}}$ and PP. Average values of sensitivity (Se), specificity (Sp) and accuracy (Acc) for the 10 iterations of learning and test carried out are presented.

\begin{tabular}{|c|c|c|c|c|c|c|c|}
\hline \multicolumn{2}{|c|}{ Variability estimation } & \multicolumn{3}{|c|}{ Learning sets } & \multicolumn{3}{|c|}{ Test sets } \\
\hline$\overline{\mathrm{CTM}}$ & $\alpha$ & $\mathrm{Se}$ & $\mathrm{Sp}$ & Acc & $\mathrm{Se}$ & $\mathrm{Sp}$ & Acc \\
\hline $\mathrm{P}_{\mathrm{al}}$ & $\mathrm{P}_{\text {dur }}$ & $79 \%$ & $92 \%$ & $86 \%$ & $93 \%$ & $82 \%$ & $87 \%$ \\
\hline$P_{n r m s}$ & $\mathrm{P}_{\mathrm{al}}$ & $92 \%$ & $96 \%$ & $94 \%$ & $81 \%$ & $93 \%$ & $87 \%$ \\
\hline$P_{\text {area }}$ & $\mathrm{P}_{\mathrm{al}}$ & $86 \%$ & $94 \%$ & $90 \%$ & $81 \%$ & $96 \%$ & $89 \%$ \\
\hline$P_{\text {dur }}$ & $\mathrm{P}_{\mathrm{al}}$ & $89 \%$ & $89 \%$ & $89 \%$ & $89 \%$ & $85 \%$ & $87 \%$ \\
\hline $\mathrm{P}_{\mathrm{ini}}$ & $\mathrm{P}_{\mathrm{al}}$ & $89 \%$ & $91 \%$ & $90 \%$ & $92 \%$ & $88 \%$ & $90 \%$ \\
\hline $\mathrm{P}_{\text {ter }}$ & $\mathrm{P}_{\text {dur }}$ & $96 \%$ & $91 \%$ & $93 \%$ & $78 \%$ & $83 \%$ & $80 \%$ \\
\hline PP & $\mathrm{P}_{\mathrm{al}}$ & $91 \%$ & $86 \%$ & $89 \%$ & $80 \%$ & $83 \%$ & $82 \%$ \\
\hline
\end{tabular}

